\title{
Freeform active mirror designed for additive manufacturing
}

\section{Szigfrid Farkas, Tibor Agócs, Carolyn Atkins, Leon Brouwers,} Joris Dufils, András Joó, György Mező, Katherine Morris, Michiel Rodenhuis, Mélanie Roulet, Hermine Schnetler, Robert Snell, Fabio Tenegi-Sanginés, Afrodisio Vega-Moreno and Bart van de Vorst

\section{Published version information:}

Citation: S Farkas et al. 'Freeform active mirror designed for additive manufacturing.' Proceedings of SPIE, vol. 11451 (2020): 114512M. Is in proceedings of: SPIE Astronomical Telescopes + Instrumentation 2020, Online Only, United States, 14-18 Dec 2020.

DOI: $\underline{10.1117 / 12.2561327}$

(C)2020 Society of Photo-Optical Instrumentation Engineers (SPIE). One print or electronic copy may be made for personal use only. Systematic reproduction and distribution, duplication of any material in this publication for a fee or for commercial purposes, and modification of the contents of the publication are prohibited.

This version is made available in accordance with publisher policies. Please cite only the published version using the reference above. This is the citation assigned by the publisher at the time of issuing the APV. Please check the publisher's website for any updates. 


\section{Freeform active mirror designed for additive manufacturing}

Farkas, Szigfrid, Agócs, Tibor, Atkins, Carolyn, Brouwers, Leon, Dufils, Joris, et al.

Szigfrid Farkas, Tibor Agócs, Carolyn Atkins, Leon Brouwers, Joris Dufils, András Joó, György Mező, Katherine Morris, Michiel Rodenhuis, Mélanie Roulet, Hermine Schnetler, Robert Snell, Fabio Tenegi-Sanginés, Afrodisio Vega-Moreno, Bart van de Vorst, "Freeform active mirror designed for additive manufacturing," Proc. SPIE 11451, Advances in Optical and Mechanical Technologies for Telescopes and Instrumentation IV, 114512M (13 December 2020); doi: 10.1117/12.2561327

SPIE. Event: SPIE Astronomical Telescopes + Instrumentation, 2020, Online Only 


\title{
Freeform active mirror designed for additive manufacturing
}

\author{
Szigfrid Farkas*a , Tibor Agócs ${ }^{\mathrm{b}}$, Carolyn Atkinss ${ }^{\mathrm{c}}$, Leon Brouwers ${ }^{\mathrm{d}}$, Joris Dufils ${ }^{\mathrm{d}}$, András Joóa

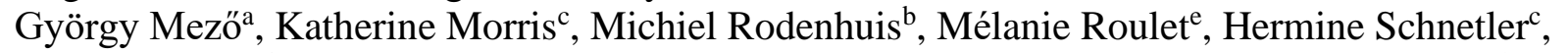 \\ Robert Snell ${ }^{\mathrm{f}}$, Fabio Tenegi-Sanginés ${ }^{\mathrm{g}}$, Afrodisio Vega-Moreno ${ }^{\mathrm{g}}$, and Bart van de Vorst $^{\mathrm{d}}$ \\ ${ }^{a}$ Konkoly Observatory, Research Centre for Astronomy and Earth Sciences, MTA Centre for \\ Excellence, 1121 Konkoly Thege Miklós út 15-17, Budapest, Hungary; ${ }^{b}$ NOVA Optical \& Infrared \\ Instrumentation Group at ASTRON, P.O. Box 2, 7990 AA Dwingeloo, the Netherlands; ${ }^{c} \mathrm{UK}$ \\ Astronomy Technology Centre (UK ATC), Blackford Hill, Edinburgh, EH9 3HJ, Scotland, UK; \\ ${ }^{\mathrm{d}}$ TNO, 5656 AE Eindhoven, the Netherlands; ${ }^{\mathrm{A}}$ Aix Marseilles Univ, CNRS, CNES, LAM, \\ Marseilles, France; ${ }^{\mathrm{f} D e p a r t m e n t}$ of Materials Science and Engineering, University of Sheffield,

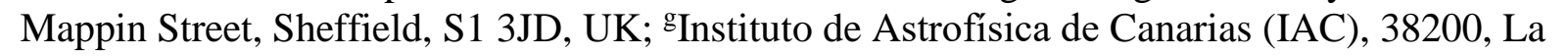 \\ Laguna, (S/C Tenerife), Spain
}

\begin{abstract}
A possible answer to the challenge brought by the construction of the next generation $40 \mathrm{~m}$-class telescopes was the case study of FAME (Freeform Active Mirror Experiment). As the new instruments typically increased in both volume and complexity, the use of highly aspherical freeform surfaces could be a great solution as these systems are built up from fewer parts and can achieve higher performance. The idea of FAME was to create a thin face sheet which is then deformed to the nominal shape utilizing actuators mounted on the back of the mirror and acting parallel to the optical surface.

The test phase of the FAME prototype revealed the complexity of the design and its sensitivity to manufacturing and assembly processes. As part of the characterization it was very difficult to predict correctly how the system behaves which is due to the several interfaces between the actuators and the face sheet.

These experiences led to the development of a new structure that eliminates the strict tolerance chain obtained from a variety of components mounted on one another. It also means that the design for conventional manufacturing technologies should be left behind, and additive manufacturing must be introduced. This paper gives a brief overview how the lessons learned from the previous development is matched with the new design approach of the same component using topology optimization, additive manufacturing of metals and post processing of 3D printed parts.
\end{abstract}

This work is funded under the OPTICON H2020 INFRAIA-2016-2017/H2020-INFRAIA-2016-1 Grant Agreement 730890 .

Keywords: active, additive manufacturing, analysis, design, freeform, generative, mirror, optimization

\section{INTRODUCTION}

In the past years there were several ideas how to build active deformable mirrors, both with actuators working perpendicular [1] and parallel to the aperture [2]. Also, the Freeform Active Mirror Experiment (FAME) [3] of OPTICON FP7 framework tried to solve the challenge of developing a high curvature freeform mirror that can be deformed to match the desired target surface in a seemingly simple way. The technology available to the team at that time was not suitable to meet all the requirements and now still there is a lot of work to do. Giving an exact solution is not possible due to the complexity of the problem, as an incredibly long iteration process and the combination of steps of a well-defined workflow is necessary to proximate the desired results.

*farkas.szigfrid@csfk.mta.hu; phone +36 1391 9346; konkoly.hu

Advances in Optical and Mechanical Technologies for Telescopes and Instrumentation IV, edited by Ramón Navarro, Roland Geyl, Proc. of SPIE Vol. 11451, 114512M

(C) 2020 SPIE · CCC code: 0277-786X/20/\$21 · doi: 10.1117/12.2561327 
During the manufacturing and assembly of the FAME demonstrator, several ideas were found to set the future development of the system. The original structure consisted of nearly 200 parts, excluding the large number of actuators necessary to create the prescribed deformation of the aperture. At this point it was obvious to replace the parts with a single unibody component to avoid the complications gained from the long-drawn tolerance chain that was caused by the parts that can be positioned to each other in very difficult and inaccurate way. The use of non-compliant bonding technology also made it clear the necessity to find an additive manufacturing technique in the future. The feasibility study of $\mathrm{H}$. Saudan [4] proved that compliant structures can be redesigned along the rules of additive manufacturing and topology optimization.

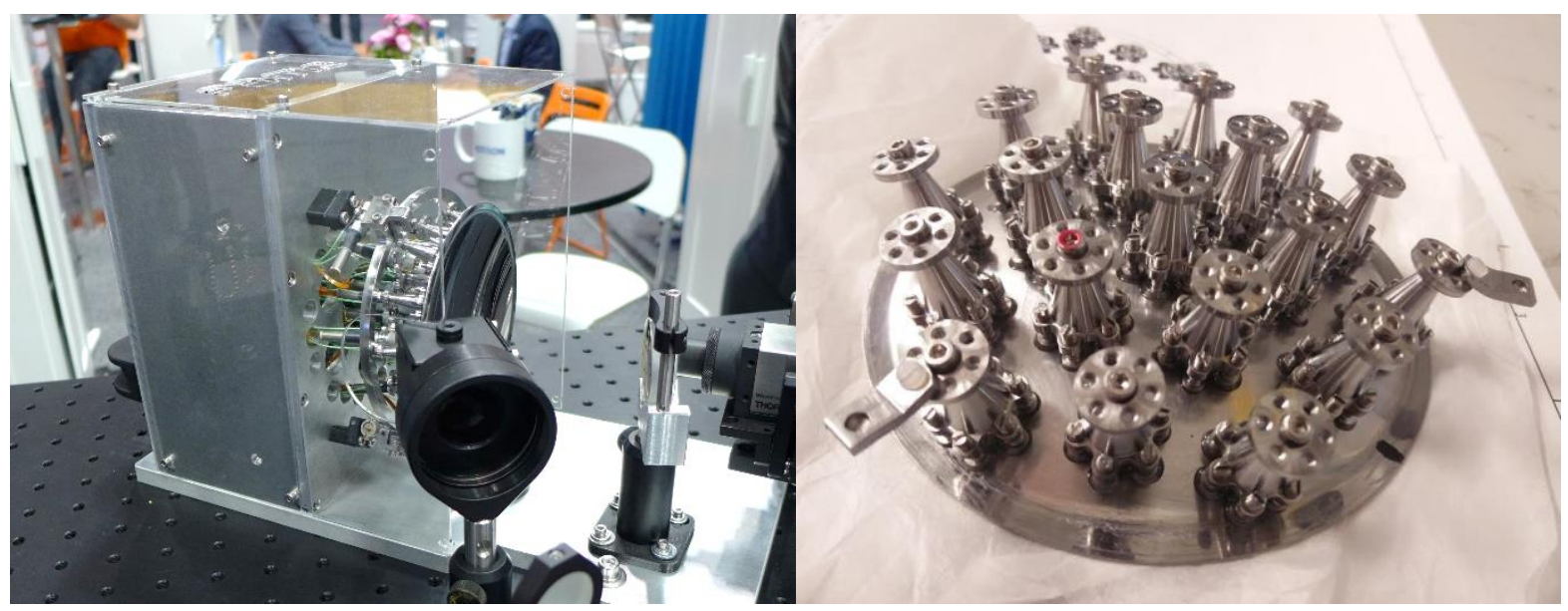

Figure 1. FAME demonstrator (left) and the modular active array bonded on the back of the face sheet (right).

The "Additive astronomy related integrated component manufacturing" (A2IM) project, an OPTICON H2020 work package, gave a perfect opportunity for the revision of the original FAME prototype specifically from design and manufacturing technology point of view. The work done so far revealed that the process is not obvious at all and control can be lost at several points. The rest of this paper tries to discuss the preliminary design method and the techniques used to develop a new FAME+ prototype candidate along the guidelines of design for additive manufacturing (DfAM), hereby giving some help for solving similar engineering problems.

\section{GUIDELINES}

\subsection{Design considerations}

The aim is to deform a well described simple initial aperture to reach the desired freeform shape within 1 um RMS, but it is not about the correction of manufacturing errors described by certain Zernikes. The initial aperture forms a simple axisymmetric shape (paraboloid or spherical) that can be manufactured with high precision and reliability by polishing or diamond turning. In our case this shape is a sphere, the required deformations are based on the departure from this shape.

An exceptionally smooth transition between the face sheet and the active array must be created to reduce the significant print-through observed during the optical tests of the FAME mirror. This variable thickness of the face sheet enables the foundation of "flexures" with different bending stiffnesses, so actuators with the same force output can be used on different mounting points. Former study of A. Jaskó [5] showed the effectiveness of active array with 126 actuators. The original actuator array of FAME consisting of 42 units on a regular arrangement could theoretically produce 4.5 um RMS deformation on the best fit sphere. However, the tested system was finally populated with only 10 actuators, the residuals were greatly increased to 16 um RMS. Despite the large number of actuators needed to fully populate the system seemed too high, it was not changed in the preliminary design stage due to the reduction of design variables. 


\subsection{Technology considerations}

Cryogenic compatibility is required to use the device for infrared applications. According to the study of W. Stautner [6], the options for 3D printable materials working under cryogenic conditions are wide, even considering polymers. Since the reduction of the number of components has high priority, the reflective surface should be integrated in the deformable structure. Therefore, materials with good machining and polishing properties are favored, the available options were already investigated by C. Atkins [7]. Choices are restricted to aluminum alloys (ie. AlSi10Mg), stainless steels (ie. PH1 or 316L) and titanium alloy (Ti6Al4V), due to the traditional way of component design based on coefficient of thermal expansion (CTE) differences. Again, to reduce the number of design variables, stainless steel is used for the computations. The chosen manufacturing technique is direct metal laser sintering (DMLS) over metal fused filament fabrication (FFF) due to FFF's poor build up quality, that might be familiar from desktop fused deposition modelling (FDM) 3D printers, such as stair stepping, substantial porosity between contour and infill lines, additionally, a loose microstructure and nonuniform shrinkage during sintering.

\section{THE PRELIMINARY DESIGN APPROACH}

\subsection{The applied generative design method}

Recently certain integrated CAE and FEA systems have shape optimization tools that can create organic generative shapes based on predefined design objectives. This way the load path can be revealed under the coverage of unnecessary material, which results complex shapes usually not suitable for conventional manufacturing techniques such as milling. These tools are often advertised as simple to use solutions for industrial demands, but they only work flawlessly until the 2.5D applications are not exceeded. As soon as more complex shapes are generated than a single extrusion the lower level CAD packages start to fail reconstructing the geometries imported from third party topology optimization software. The proper clean up and conversion to editable format of resultant STL files required more resources than previously expected.

Despite the complexity of the task we tried to keep the number of design software used maintain on a minimum level. The workflow applied is shown in Figure 2.

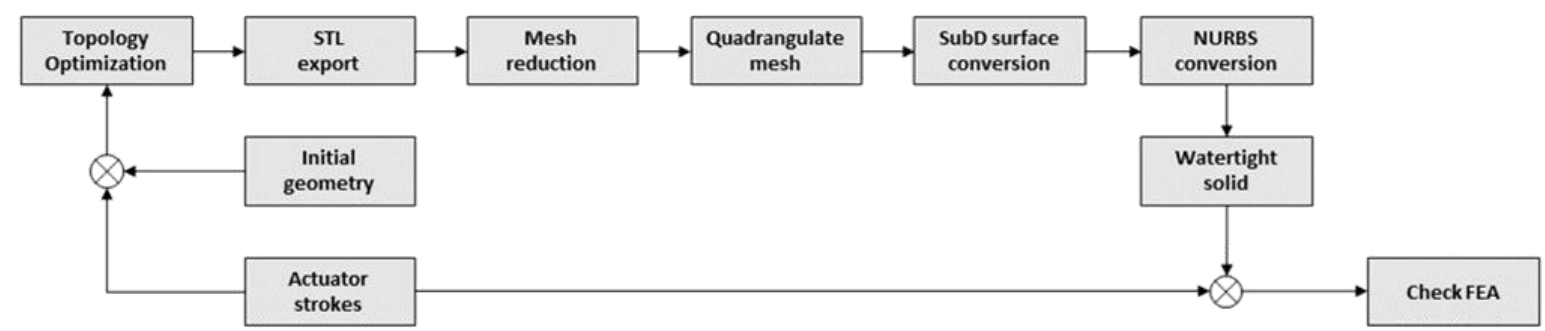

Figure 2. Workflow diagram.

Our option for mechanical design was Autodesk Inventor. Although this software offers its own simplified solution called "shape generator", Inventor was only used for creating the initial geometry that has the features of reflective surface, mounting points of actuators and the mounts of the mirror itself. These entities must remain intact during the optimization process, a certain part of the surrounding material is removed by the algorithm.

The actuator strokes required to generate the target shape of the aperture are computed using ANSYS. The effects of each actuator are simulated with the same stroke value, the deformation of the aperture is exported in tabular format. These raw influence functions computed on the finite element mesh are then converted to a rectangular matrix form that can be handled by the improved version of the optical performance simulation code developed in Python [8]. This code is responsible for calculating the strokes on each actuator locations. The workflow until this point is familiar from the former process. The response is checked using ANSYS, it gives the requisite for the ANSYS Topology Optimization module. After setting the design objectives, including additive manufacturing constraints, the generated geometry is ready for 
export in a triangular surface mesh format (STL). There is an option to export as smooth mesh, but it will refine the finite element residues only, which might be enough in many cases. In less fortunate situations this mesh needs a fair amount of post-processing where options diverge into two ways.

The more labor-intensive way is remodeling the surface in the preferred CAD application. Here Inventor offers its own freeform modeling tool, of which limitations can easily be reached. The other way is the direct modification and conversion of the resultant surface mesh. Subdivision (SubD) modelling plays a key role in this method and the weapon of choice for the task is McNeel's Rhinoceros, a non-uniform rational B-spline (NURBS) modeling application, as a reliable and cheap solution. First step is mesh reduction. In this example the mesh polygon count was reduced by nearly $1 / 1000$, so a very coarse, boxy shape was created. It is followed by the conversion of triangular mesh to quadrilateral mesh. Probably this is the most time-consuming part of the process, as the merging of neighboring triangles is not always possible automatically or neatly. The SubD conversion is used to interpolate a smooth shape from the rough blocks, it is done by the Catmull-Clark algorithm. The simpler the block mesh, the smoother the surface is. One more conversion is needed as the latest shape cannot be directly modified as a surface or solid model in any of the applications used in this study. The importance of quadrilateral mesh is shown in Figure 3 e), when the SubD to NURBS conversion is done, as all triangles are divided into three quads, meaning a badly prepared mesh can result in a too large file to handle, but at least it can be manipulated in engineering CAD applications when necessary. These steps allow for a check and refinement of the topology optimized geometry. Finally, the new shape has minor differences compared to the original STL file, the most significant improvement is the elimination of sharp edges along the initial and optimized domains, as seen in Figure 4.

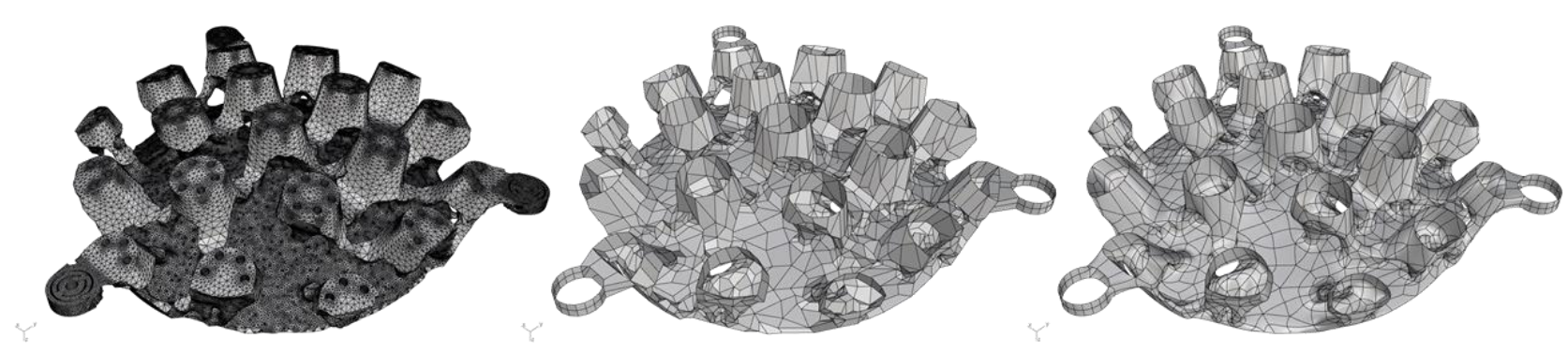

a)

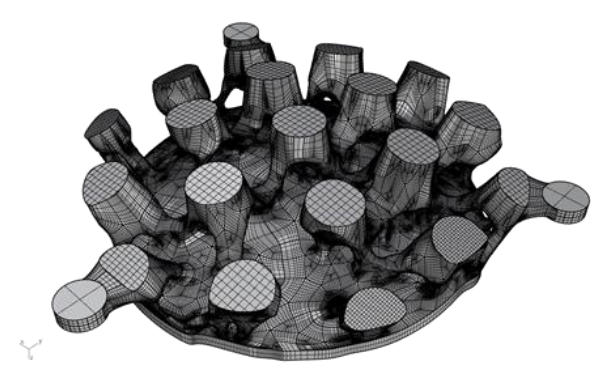

d) b)

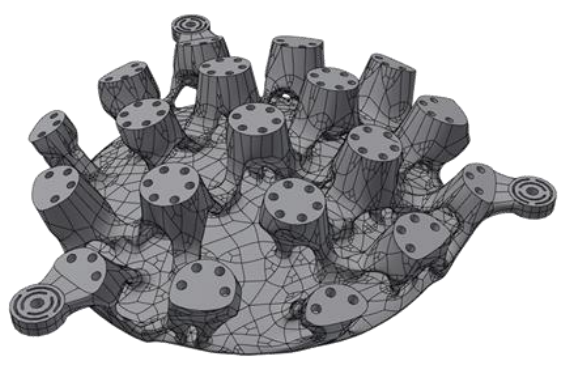

e)

Figure 3. Evolution of the model in different stages: a) the original STL mesh, b) the reduced and mostly quadrangulated mesh, c) the subdivision surface and d) the watertight NURBS polysurface (solid body) closed by basic features and planar segments, e) finalized solid geometry. 


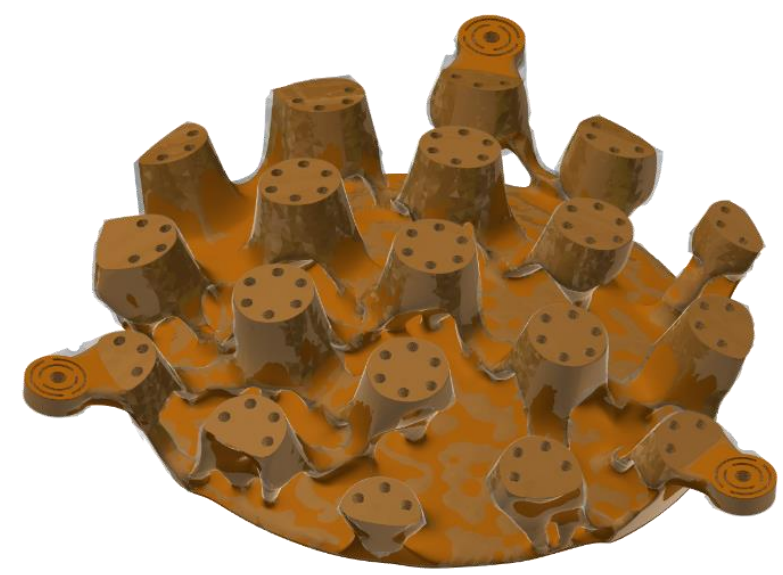

Figure 4. Differences in shape and surface structure between the original STL file (translucent grey) and the final smoothed solid geometry (orange).

Modelling the initial geometry is usually straight forward: a block with exclusion regions is enough to run the simulation and determine the elements in the unnecessary volumes. After the evaluation of the influence functions the resultant actuator strokes showed extremely high values, meaning that the displacements used to generate the influence functions have no effect on the shape of the aperture. Investigating the finite element model and the results of the simulation, the actuator effects were local only, deforming the near vicinity of the actuator mounts. So, in this case some intuitive approach was needed to converge the expected optimal result, the evolution of initial geometries is shown in Figure 5. Even the attempts with 20 and $10 \mathrm{~mm}$ minimum thicknesses were too stiff. A reduction of thickness to $1.5 \mathrm{~mm}$, which was obtained from one of the optimized shapes, a print through was observed on aperture, so the playground of the optimization algorithm was eliminated.

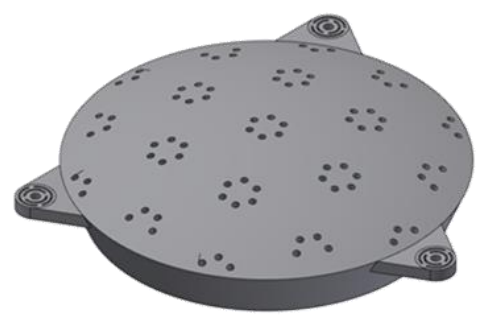

a)

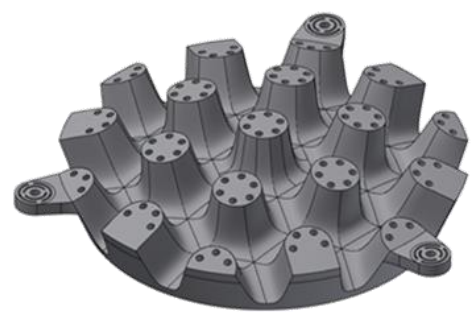

c)

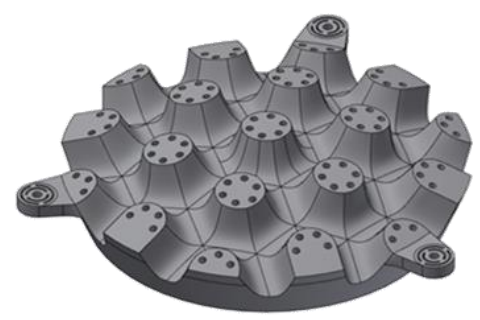

b)

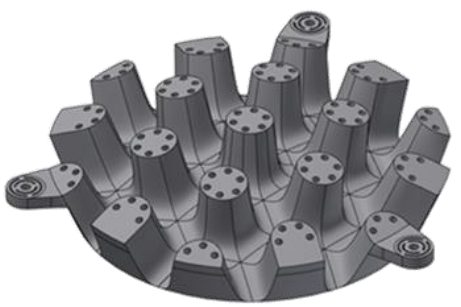

d)

Figure 5. Development of the appropriate initial geometries: a) solid disc of $20 \mathrm{~mm}$ thickness, b) $10 \mathrm{~mm}$ thickness between active array nodes, c) $5 \mathrm{~mm}$ thickness between active array nodes, d) $1.5 \mathrm{~mm}$ thickness between active array nodes. 


\subsection{Optical performances}

At this stage optical performance analysis had a key role in finding the proper initial geometry. The method is based on the determination of influence functions using finite element simulations. To create these influence functions, $0.05 \mathrm{~mm}$ actuator displacements are simulated on each location, considering the presence of all actuators. Beside the nominal aperture diameter of $80 \mathrm{~mm}$ (Figure 6 and Figure 7), the full free surface area, a diameter of $100 \mathrm{~mm}$, was also investigated (Figure 8 and Figure 9). The required shape can be approximated with the linear combination of influence functions and the residuals can be obtained. It was important to investigate the calculated actuator displacements needed to create the generated shape. These values are defined as multipliers of influence functions, so the exact movements can be obtained easily. The proper initial geometry works with realistic actuator displacements, that preferably do not exceed the order of magnitude of $\pm 1 \mathrm{~mm}$. As an example, the first candidate required $\pm 20 \mathrm{~mm}$ actuator strokes (Figure 7), which is physically not feasible. Fine tuning of the required actuator displacements can be done by the modification of aperture diameter, but it is not possible while having an exact specification.

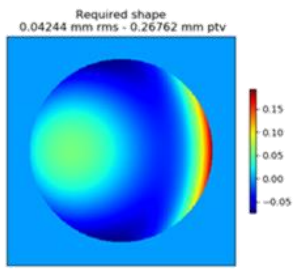

a)

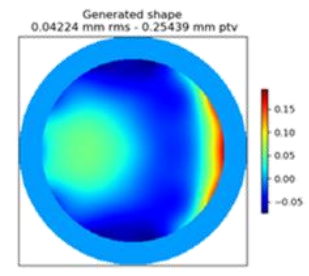

b)

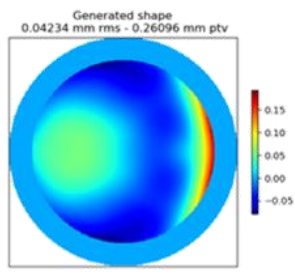

c)

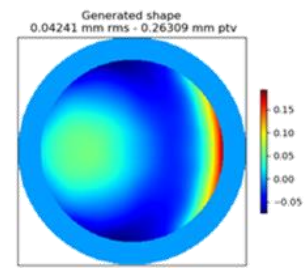

d)

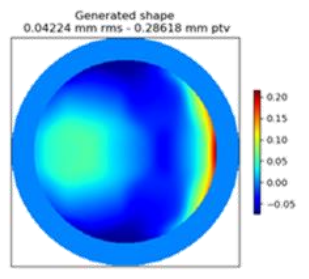

e)

Figure 6. Performance of initial geometries measured on the nominal aperture: a) target shape, b) solid disc, c) $10 \mathrm{~mm}$ thickness between active array nodes, d) $5 \mathrm{~mm}$ thickness between active array nodes, e) $1.5 \mathrm{~mm}$ thickness between active array nodes.
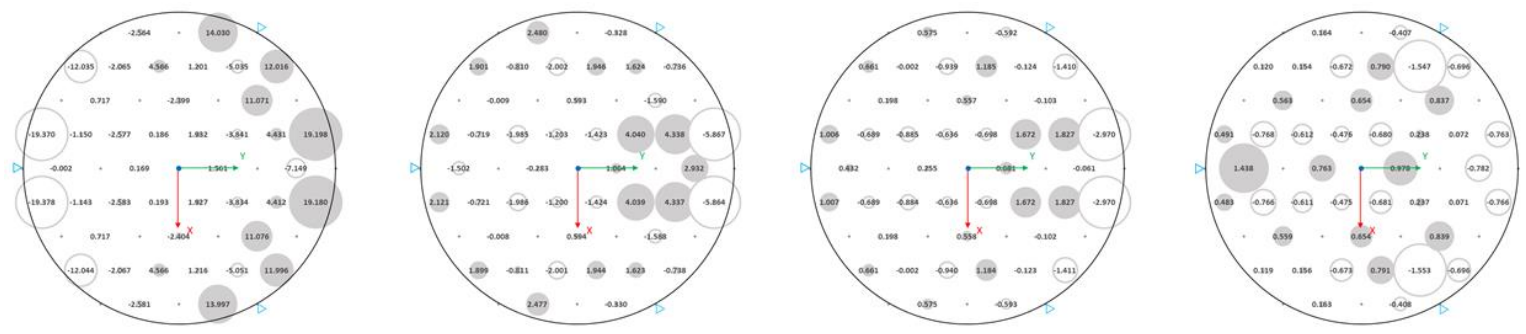

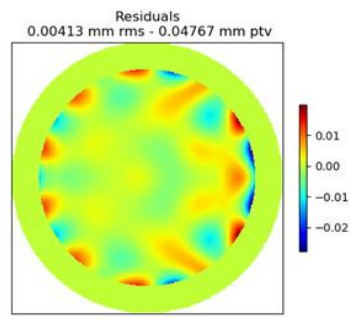

a)

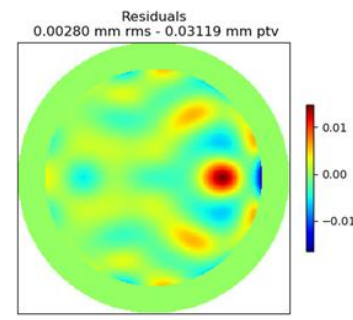

b)

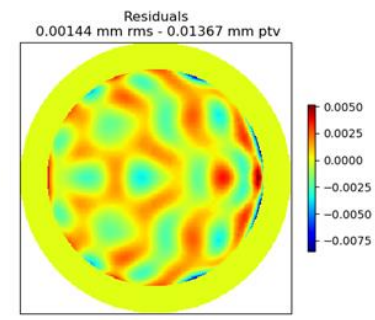

c)

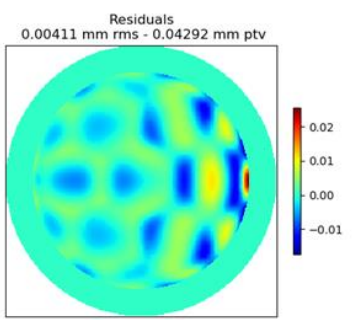

d)

Figure 7. Required actuator displacements (top) and residuals (bottom) of generated shapes measured on the nominal aperture: a) solid disc, b) $10 \mathrm{~mm}$ thickness between active array nodes, c) $5 \mathrm{~mm}$ thickness between active array nodes, d) 1.5 $\mathrm{mm}$ thickness between active array nodes. 


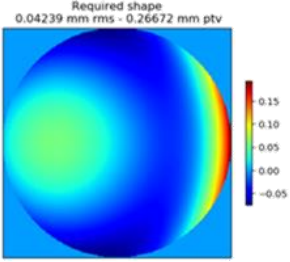

a)

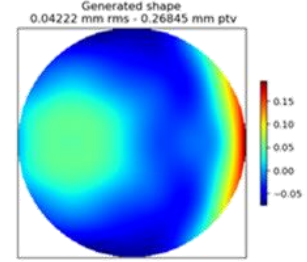

b)

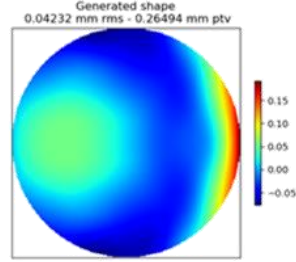

c)

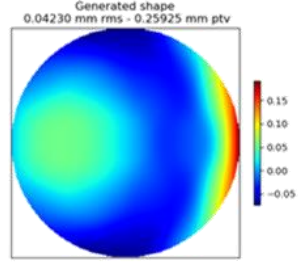

d)

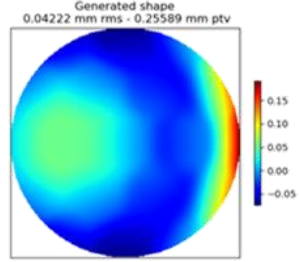

e)

Figure 8. Performance of initial geometries measured on the free surface: a) target shape, b) solid disc, c) 10 mm thickness between active array nodes, d) $5 \mathrm{~mm}$ thickness between active array nodes, e) $1.5 \mathrm{~mm}$ thickness between active array nodes.
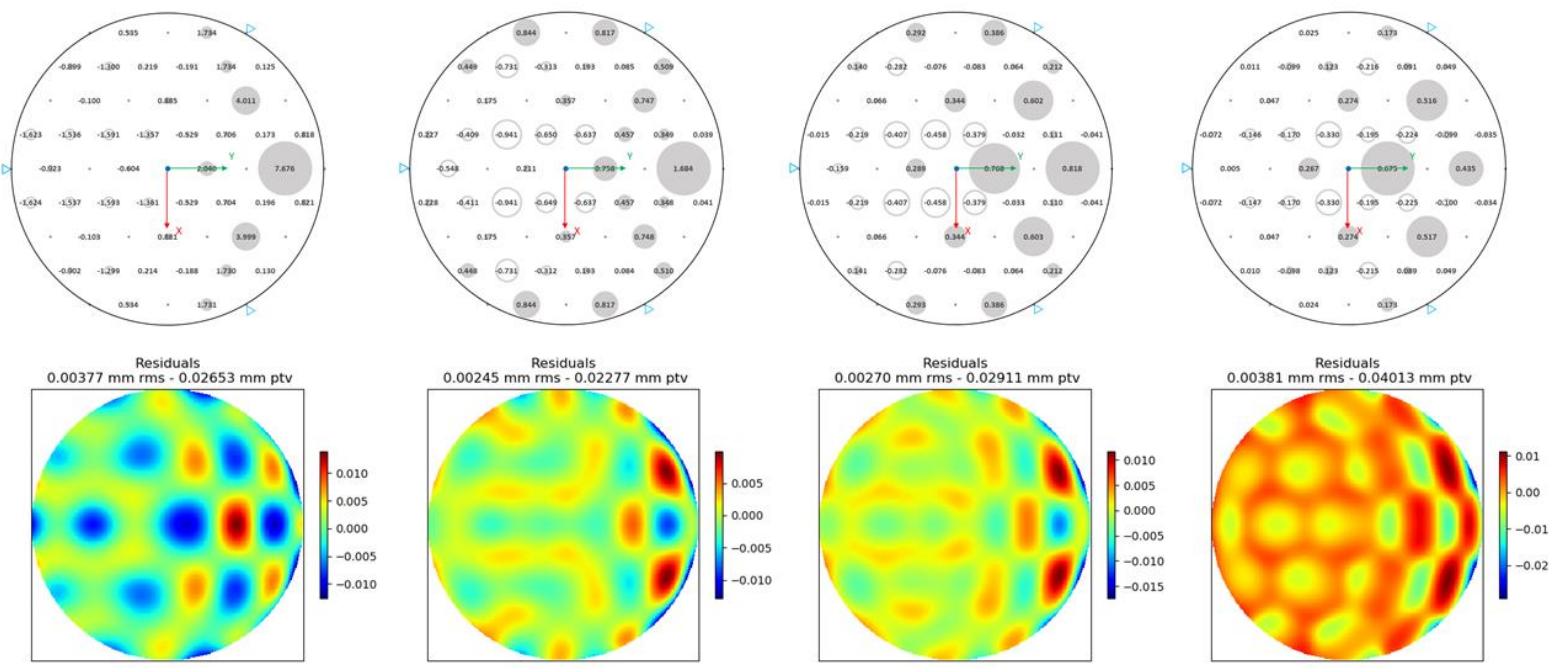

a)

b)

c)

d)

Figure 9. Required actuator displacements (top) and residuals (bottom) of generated shapes measured on the free surface: a) solid disc, b) $10 \mathrm{~mm}$ thickness between active array nodes, c) $5 \mathrm{~mm}$ thickness between active array nodes, d) $1.5 \mathrm{~mm}$ thickness between active array nodes.

When comparing the generated shapes, the lower RMS of residuals was the selection criteria, and the absence of print through was favorable. In case of the thinnest candidate, a significant print through can be seen, that cannot be eliminated by the optimization due to lack of material on sensitive regions.

\subsection{Post-manufacturing options}

There are countless ways to determine print orientation, but considering an easier post-manufacturing process, the two obvious options are investigated, where the printing direction coincides with the rotation axis of the aperture. These two options are shown in Figure 10, considering the necessary post-machining processes to guarantee the required optical surface quality.

An early idea proposed to make use of the AM support under cantilever surfaces of the model, as shown in the right of Figure 10. Since the top layers of AM parts are finished in a better surface quality than the bottom sides, polishing can be done on a smoother surface. This way the supports can be used as a post-machining jig and can be designed to withstand the pressure caused by the polishing tool. Its drawback is shown when finishing the mounting features, as the optical quality surface must be supported to bear the loads of milling, threading, and reaming. 
The other method, that was finally chosen, tries to solve the problem other way around. As left set of Figure 10 shows, the mounting features are finished before the optical surface is fabricated. A stiffening jig is still needed later to support the thin face sheet, which can be also provided using AM tools. The advantage of this approach is leaving the deformable face intact as there is no reflective area to be damaged and the AM support give enough stiffness to create the mounting features, but the polishing jig must be designed carefully not to over constrain the mirror.

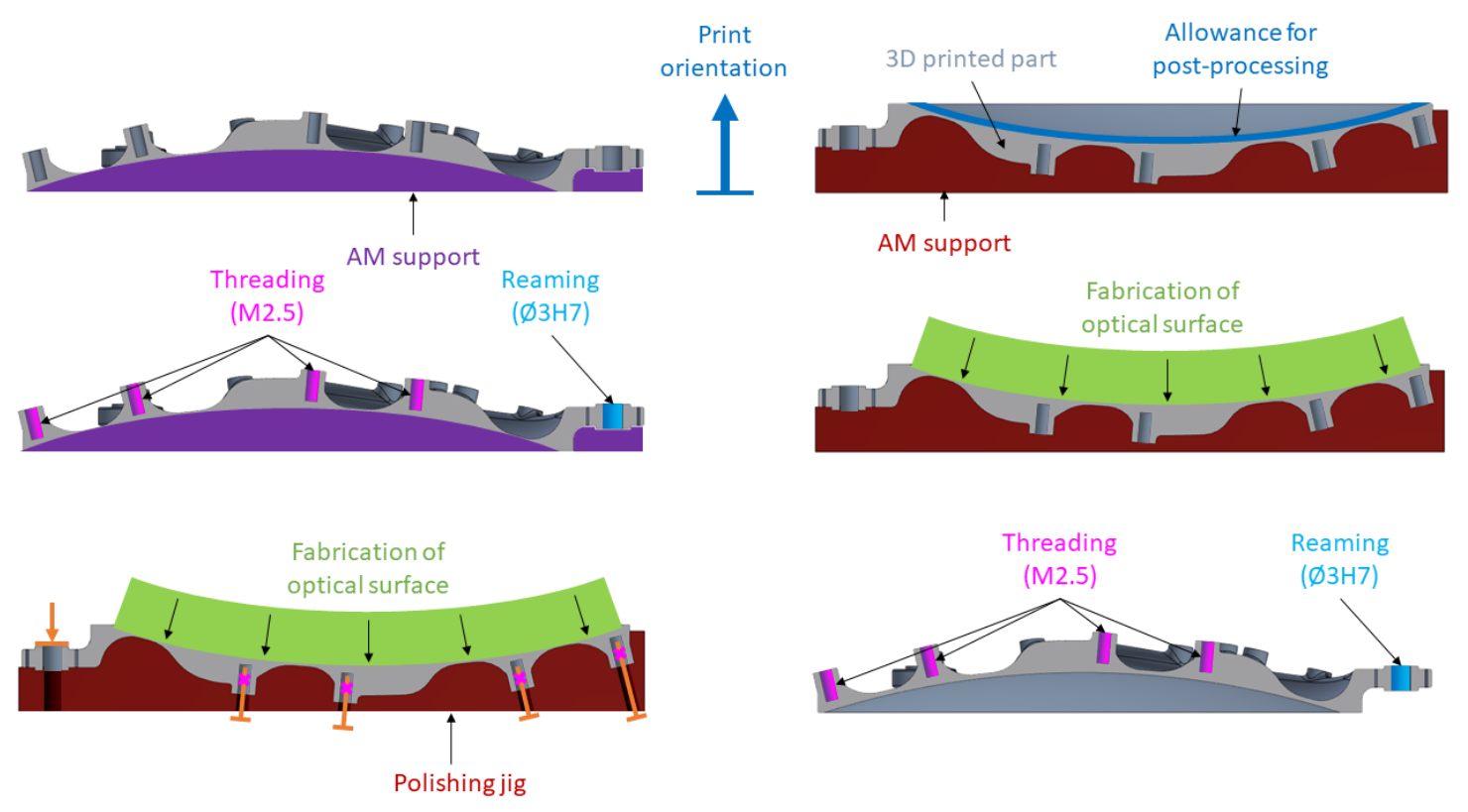

Figure 10. Schemes of possible post-machining methods. Aperture facing down during build up process and mounting features as final layers on left, aperture facing up as final layers on right.

\section{SUMMARY}

Additive manufacturing is often called as the "Holy Grail of manufacturing", but the studies published so far do not always give proper help to solve specific problems, especially in the field of astronomical instrumentation. A2IM, an OPTICON H2020 work package is intended to fill this gap by means of an engineering cookbook for certain AM applications.

The workflow described in this study was defined to post-process the resultant geometries of shape optimization algorithms that are available in some finite element analysis applications and integrated CAE solutions. The main point of the technique is to reduce the mesh polygon count of complex STL geometry which is then further processed by a Catmull-Clark algorithm that generates a smooth continuous subdivision surface. Refinement of the result can be done in the mesh reduction phase of the process. For demonstration purposes the FAME+ prototype, part of another OPTICON H2020 work package is used. A system full of design variables, such as FAME, makes the solution difficult to converge. Although this study does not give a quantitative result to the raised design problem, it gives an acceptable workflow solution for further development and the loop can be closed on analysis.

The method can be addressed for the faster and direct post-processing of future topology optimization challenges and can be useful part of the engineering cookbook. In view of the range of AM materials, the available manufacturing technologies, both additive and subtractive, the precise definition of initial geometries and optimization goals helps to get one step closer to the original objectives of FAME. 


\section{ACKNOWLEDGEMENT}

This work is funded under the OPTICON H2020 INFRAIA-2016-2017/H2020-INFRAIA-2016-1 Grant Agreement 730890 .

\section{REFERENCES}

[1] Trines, R., Janssen, H., Paalvast, S., Teuwen, M., Brandl, B., and Rodenhuis, M. "A cryogenic 'set-and-forget' deformable mirror", Proc. SPIE 9912, Advances in Optical and Mechanical Technologies for Telescopes and Instrumentation II, 99121B (2016)

[2] Steeves, J., Redding, D., Wallace, J. K., Lawrence, C., Gaier, T., Bartman, R., Mikhail, R., Cavaco, J., and Vayda, J. "Active mirrors for future space telescopes", Proc. SPIE 10706, Advances in Optical and Mechanical Technologies for Telescopes and Instrumentation III, 1070615 (2018)

[3] Aitink-Kroes, G., Agócs, T., Miller, C., Black, M., Farkas, S., Lemared, S., Bettonvil, F., Montgomery, D., Marcos, M., Jaskó, A., van Duffelen, F., Challita, Z., Fok, S., Kiaeerad, F., Hugot, E., Schnetler, H., and Venema, L. "FAME: freeform active mirror experiment", Proc. SPIE 9912, Advances in Optical and Mechanical Technologies for Telescopes and Instrumentation II, 99121I (2016)

[4] Saudan, H., Kiener, L., Perruchoud, G., Kruis, J., Vaideeswaran, K., Dadras, M., Cochet, F., and Liberatoscioli, S. "Compliant mechanisms and space grade product redesign based on additive manufacturing", Proc. SPIE 10706, Advances in Optical and Mechanical Technologies for Telescopes and Instrumentation III, 107062T (2018)

[5] Jaskó, A., Aitink-Kroes, G., Agócs, T., Venema, L., Hugot, E., Schnetler, H., and Bányai, E. "Active array design for FAME: Freeform Active Mirror Experiment", Proc. SPIE 9151, Advances in Optical and Mechanical Technologies for Telescopes and Instrumentation, 91512M (2014)

[6] Stautner, W., Vanapalli, S., Weiss, K.-P., Chen, R., Amm, K., Budesheim, E., and Ricci, J. "The scope of additive manufacturing in cryogenics, component design, and applications," IOP Conference Series: Materials Science and Engineering, 278(1), [012134], (2017)

[7] Atkins, C., Brzozowski, W., Dobson, N., Milanova, M., Todd, S., Pearson, D., Bourgenot, C., Brooks, D., Snell, R., Sun, W., Cooper, P., Alcock, S. G., and Nistea, I.-T., "Additively manufactured mirrors for CubeSats," Proc. SPIE 11116, Astronomical Optics: Design, Manufacture, and Test of Space and Ground Systems II, 1111616 (16 September 2019)

[8] Laslandes, M., Hugot, E., Ferrari, M., Hourtoule, C., Lopez, C., and Chazallet, F. "Mirror actively deformed and regulated for applications in space: design and performance," Optical Engineering 52(9), 091803 (2013) 